\title{
Adultos mayores institucionalizados en Chile: ¿Cómo saber cuántos son?
}

\author{
Pedro Paulo Marín L1, José Miguel G uzmán $M^{2}$, \\ Alejandra Araya $\mathbf{G}^{3}$. \\ Estimation of the number of \\ institutionalized elderly in Chile
}

Background: Elderly people ( $>60$ years) in Chile represented $11.4 \%(\mathrm{n}=1.717 .478)$ of the total population in 2002. The group with disabilities or mental problems is increasing and there is no reliable information about the number of institutionalized elderly subjects. Aim: To estimate the number of elderly people living in residences for long term care and their and main characteristics. Patients and methods: Chilean Census does not provide exact information about institutional care, therefore we developed a proxy»indicator of the percentage of institutionalized elderly (those living in «collective residences with more than 5 elderly persons and in which they represent more than $25 \%$ of the residentss. This proxy has a R2 $=0.9859$ with the true value of institutionalized persons for those Latin-American countries with exact value in census data at CELADE. Results: Using the proxy we found that institutionalized elderly population had increased from 14,114 (1992) to 26,854 (2002) and is projected to reach 83,500 (2025). In 2002, there were 1.668 institutions (37.4\% informal care). In the Metropolitan Area, there were 804 institutions (14.178 elderly persons) and $40.3 \%$ of these were registered at the Ministry of Health. The proportion of institutionalized elderly subjects was $1.56 \%$ of the total elderly population; this proportion increased from $0.87 \%$ in subjects $60-74$ years old to $2.5 \%$ among subjects aged $75-84$ years and $6.1 \%$ in subjects 85 years old and over. Among subjects living in institutions, 60.9 were women, $21 \%$ were married, 35\% were single, approximately $50 \%$ receive a pension and around $15 \%$ were handicapped. Conclusions: Institutional care affects a small percentage of elderly population, but it will increase in the near future. The main characteristics of institutionalized elderly subjects are not well known. We propose to create a formal Registry of these institutions and to include Nursing Homes and hospitals in type of housing of future Censuses (Rev Méd Chile 2004; 132: 832-8).

(Key Words: Aged; Housing for the elderly; Institutionalization; Proxy)

Recibido el 23 de diciembre de 2003. Aceptado el 6 de abril de 2004.

${ }^{1}$ Centro de Geriatría y Gerontología PUC, Departamento de Medicina Interna. ${ }^{3}$ Escuela de Enfermería, Facultad de Medicina, Pontificia Universidad Católica de Chile.

${ }^{2}$ Centro Latinoamericano de Demografía - CELADE, División de Población CEPAL.

aEnfermera Universitaria.

Correspondencia a: Dr. Pedro Paulo Marín L. Programa de Geriatría, Facultad de Medicina, Pontificia Universidad Católica de Chile. Casilla 114-D, Santiago, Chile.

E-mail: ppmarin@med.puc.cl 
$\mathrm{D}$ ebido al rápido incremento del número de personas mayores de sesenta años (adultos mayores, $\mathrm{AM}$ ) en el mundo y sobre todo de aquellos más envejecidos, es que la organización y prestación de servicios de salud y en especial la «atención a largo plazo»toma cada vez más relevancia desde el punto de vista político y se transforma, además, en una necesidad socio-familiar. Los estudios internacionales demuestran que los individuos más ancianos aumentan cada vez con más rapidez y que concentran aquellos con más limitaciones funcionales que afectan su independencia en el diario vivir, por lo que requieren una valoración geriátrica integral y asistencia especial; además, se incrementan aquellos AM con trastornos mentales, cognitivos y del comportamiento, lo que hace más engorroso su cuidado a largo plazo ${ }^{1-4}$.

El desafío actual, para nuestros gobiernos, es brindar atención en forma moderna y adecuada a las necesidades del nuevo usuario de los servicios de salud, que es la persona mayor. Ello requiere establecer un abanico de servicios y cuidados de salud que se empleen tanto para los AM que estén sanos, como para aquellos con enfermedades agudas, limitaciones funcionales que necesiten rehabilitación y enfermedades crónicas más discapacitantes o con problemas mentales, que requieren cuidados prolongados ${ }^{1-7}$.

Este «cuidado de largo plazo» es requerido por un porcentaje pequeño de la población de AM, estimándose en menos de $8 \%$ de ellos. Pero cada vez son más necesarios, ya que la población de $\mathrm{AM}$, a su vez, se está envejeciendo y se observan cambios socio-familiares en las sociedades actuales (familias y viviendas más pequeñas, mujeres trabajando fuera del hogar, etc.). Este tipo de pacientes necesita cuidado durante las 24 horas y ello se puede realizar en las propias viviendas 0 en instituciones. Es común observar que la persona cuidadora de la comunidad (familiar o contratada), realiza este trabajo en el domicilio con abnegación, pero a veces sucumbe o no hay una red social de apoyo, por lo que el paciente es derivado a una residencia de ancianos»-11.

En Chile no existe un registro fiable del número de instituciones», pero sí hay una reglamentación del Ministerio de Salud al respecto. Nuestro grupo ha estado interesado en este tema y ha publicado algunos trabajos al respecto ${ }^{3,12-14}$ por lo que decidimos intentar cuantificar el núme- ro de residencias existentes en nuestro país y conocer algunas de las características de los AM institucionalizados.

\section{MATERIAL Y MÉTODO}

En este trabajo descriptivo, se usó la información de las bases de datos censales de los países latinoamericanos disponibles en el Centro Latinoamericano de Demografía (CELADE/División de Población de CEPAL). En todos está la vivienda definida como sparticular»o «colectiva»(hospitales, asilos, etc) y en algunos países está explicitado que el AM reside en una institución.

En la boleta del Censo chileno no se describe el tipo de vivienda colectiva, por lo cual no es posible determinar exactamente el número de los AM institucionalizados, definidos acá como aquellos que viven en residencias de ancianos o que permanecen por largo plazo en hospitales. Para aproximarse, se definió un indicador proxi»como siviendas colectivas en que habitan cinco o más adultos mayores y en los que éstos constituyen al menos el 25\% del total de residentes». Con esta definición, para aquellos países latinoamericanos que sí cuentan con la variable definida y registrada en la Boleta Censal, se realizó un análisis de regresión entre este proxi»y el número que efectivamente vive en hogares de ancianos o se encuentra en hospitales, encontrándose una alta correlación $\left(R^{2} 0,9859\right)$ y una pendiente muy cercana a 1, lo que permite usarlo directamente como un indicador fiable. A partir de la recta de regresión estimada, se procedió a obtener para Chile la estimación del número y porcentaje de AM institucionalizados y del número de residencias (denominadas residencias colectivas formales»). Adicionalmente, se usó el mismo proxi»con las viviendas particulares, mediante el cual se estimaron las que se han denominado residencias colectivas informales».

El procesamiento de los datos y el análisis estadístico, se efectuó con el programa REDATAM aplicado a la base de microdatos censales disponibles en CELADE.

\section{RESULTADOS}

En Chile habría un total de 1.668 residencias de ancianos», usando el proxi»en el censo 2002; de 
éstos $624(37,4 \%)$ corresponden a la definición de residencias colectivas informales»y 1.044 a la de residencias colectivas formales». En la Región Metropolitana (RM) habrían 804 residencias $(48,2 \%$ del total) siendo 520 formales y 284 $(35,3 \%)$ informales. Las Oficinas de Profesiones Médicas del Ministerio de Salud tenían autorizadas, hasta octubre de 2003, a 324 (área norte 16, sur oriente 15 , oriente 202 , occidente 28 y sur 63) o el 40,3\%. En la Tabla 1, se describen las características de los 14.178 AM posiblemente institucionalizados en la RM (52,8\% del total), destacando que la mayoría son mujeres y que la institucionalización aumenta con la edad.

Aplicando el mismo sproxi» al Censo de 1992 de Chile, se encuentran 14.114 personas AM posiblemente institucionalizadas $(1,08 \%)$, cifra que en el Censo de 2002 sube a un total de 26.854 adultos mayores, o sea 12.740 personas más, en diez años. Si se supone una tasa de crecimiento de la población similar a la observada entre ambos Censos y se proyectan las cifras, da como resultado que pudieran haber $83.500 \mathrm{AM}$ institucionalizados para el año 2025.

En la Tabla 2 están los resultados obtenidos con el Censo de $2002^{15}$, analizados por rango de

Tabla 2. Adultos mayores posiblemente institucionalizados en Chile: por edad y tipo de residencias

\begin{tabular}{|lccccc|}
\hline $\begin{array}{l}\text { Edad } \\
\text { (años) }\end{array}$ & $\begin{array}{c}\text { Población } \\
\text { total } \\
\mathrm{n}\end{array}$ & $\begin{array}{c}\text { Institucio- } \\
\text { nalizados } \\
\mathrm{n}\end{array}$ & $\begin{array}{c}\text { Institucio- } \\
\text { nalizados } \\
\%\end{array}$ & $\begin{array}{c}\text { AM en } \\
\text { Residencias } \\
\text { colectivas formales } \\
\%\end{array}$ & $\begin{array}{c}\text { AM en } \\
\text { Residencias } \\
\text { colectivas informales } \\
\%\end{array}$ \\
\hline $60-64$ & 499.902 & 2.981 & 0,59 & 75,5 & 24,5 \\
$65-69$ & 399.287 & 3.357 & 0,84 & 78,7 & 21,3 \\
$70-74$ & 348.118 & 4.606 & 1,32 & 84,9 & 15,1 \\
$75-79$ & 219.331 & 4.354 & 1,98 & 89,8 & 7,2 \\
$80-84$ & 137.154 & 4.663 & 3,39 & 92,4 & 5,6 \\
$85-90$ & 75.130 & 3.938 & 5,24 & 94,4 & 8,6 \\
$>90$ & 38.556 & 2.955 & 7,66 & 91,4 & 3.401 \\
n total & 1.717 .478 & 26.854 & $\bar{x} 1,56 \%$ & 23.453 & \\
\hline
\end{tabular}

Tabla 1. Características generales de los 14.178 adultos mayores de sesenta años posiblemente institucionalizados en la Región M etropolitana (RM)

\begin{tabular}{|lccc|}
\hline & $\begin{array}{c}\text { Total } \\
\text { residencias RM } \\
\mathrm{n}=804\end{array}$ & $\begin{array}{c}\text { Residencias } \\
\text { colectivas formales } \\
\mathrm{n}=520\end{array}$ & $\begin{array}{c}\text { Residencias } \\
\text { colectivas informales } \\
\mathrm{n}=284\end{array}$ \\
\hline $\mathrm{N}^{\circ}$ institucionalizados & 14.178 & 12.632 & 1.546 \\
Mujeres & 9.470 & 8.560 & 910 \\
$60-69$ años & 2.933 & 2.290 & 643 \\
$70-79$ años & 4.442 & 3.935 & 507 \\
$\geq 80$ años & 6.803 & 6.407 & 396 \\
Casado & 2.854 & 2.236 & 618 \\
Soltero & 4.683 & 4.241 & 442 \\
Viudo & 5.416 & 5.098 & 318 \\
Sabe leer y escribir & 12.490 & 11.038 & 1.452 \\
Sin discapacidad & 9.615 & 8.256 & 1.359 \\
Sólo lisiado/parálisis & 1.766 & 1.695 & 71 \\
Sólo deficiencia mental & 1.792 & 1.742 & 50 \\
Jubilado o rentista & 6.542 & 5.963 & 579 \\
Incapacitado para trabajar & 2.697 & 2.632 & 65 \\
\hline
\end{tabular}


edad y según el tipo de residencia. Observamos que la posibilidad de vivir institucionalizado en promedio es de $1,56 \%$, pero este porcentaje claramente aumenta con la edad: menos del uno $(0,87 \%)$ entre los 60 y 74 años, sube a $2,5 \%$ entre 75 y 84 años, y a 6,1\% en aquellos de 85 años y más. También, encontramos que las personas de mayor edad tienden a vivir en Residencias Colectivas Formales (de 75,5\% de aquellos entre 60 y 64 años sube sobre el $90 \%$ en los mayores de 75 años). Por el contrario, se observa que a más edad hay un menor porcentaje que vive en Residencias
Colectivas Informales: un quinto $(20,3 \%)$ de los AM entre 60 a 74 años, uno en diez de los entre 75 a 84 y baja a 7\% en el grupo de más de 84 años. También destaca, con respecto al total de chilenos de 90 años y más que sólo el 7,6\% estaría viviendo institucionalizado.

En la Tabla 3, se describen las características generales de los adultos mayores de 60 años en Chile ( $\mathrm{n}=1.717 .478)$ y de los 26.854 AM posiblemente institucionalizados. Con respecto a estos últimos, observamos que la gran mayoría (87\%) vive en Residencias Colectivas Formales. Del total

Tabla 3. C aracterísticas generales de los adultos mayores chilenos y de los 26.854 posiblemente institucionalizados

\begin{tabular}{|c|c|c|c|c|}
\hline Variable & Indicador & $\begin{array}{c}\text { Población } \\
\text { Total } \geq 60 \text { años } \\
n\end{array}$ & $\begin{array}{c}\text { AM residencias } \\
\text { colectivas } \\
\text { informales } \\
n\end{array}$ & $\begin{array}{c}\text { AM residencias } \\
\text { colectivas } \\
\text { formales } \\
n\end{array}$ \\
\hline Población total & & 1.717 .478 & 3.401 & 23.453 \\
\hline \multirow[t]{3}{*}{ Estado civil actual } & Casado & 890.565 & 1.267 & 4.435 \\
\hline & Soltero & 207.395 & 1.126 & 8.259 \\
\hline & Viudo & 453.468 & 669 & 8.818 \\
\hline Sexo & Mujeres & 959.429 & 1.967 & 14.386 \\
\hline Alfabetismo & Sabe leer y escribir & 1.486 .726 & 3.069 & 19.332 \\
\hline Lisiado/parálisis & Sí & 65.815 & 178 & 3.876 \\
\hline \multirow[t]{10}{*}{ Discapacidad } & Sin discapacidad & 1.572 .250 & 2.960 & 15.214 \\
\hline & Sólo ceguera & 20.385 & 48 & 672 \\
\hline & Sólo sordera & 37.015 & 100 & 533 \\
\hline & Sólo mudez & 1.323 & 3 & 80 \\
\hline & Sólo lisiado/parálisis & 61.552 & 169 & 3.309 \\
\hline & Sólo deficiencia mental & 18.397 & 106 & 3.370 \\
\hline & 2 discapacidades & 7.475 & 18 & 668 \\
\hline & 3 discapacidades & 840 & 0 & 76 \\
\hline & 4 discapacidades & 120 & 3 & 17 \\
\hline & 5 discapacidades & 108 & 1 & 2 \\
\hline \multirow[t]{2}{*}{ Situación laboral } & Jubilado o rentista & 711.303 & 1.350 & 11.311 \\
\hline & Incapacitado para trabajar & 44.590 & 140 & 4.421 \\
\hline \multirow[t]{9}{*}{ Religión } & Católica & 1.321 .427 & 2.635 & 19.092 \\
\hline & Evangélica & 233.877 & 299 & 1.782 \\
\hline & Testigo de Jehová & 18.374 & 30 & 94 \\
\hline & Judaica & 3.424 & 1 & 138 \\
\hline & Mormón & 11.222 & 5 & 62 \\
\hline & Musulmana & 330 & 3 & 4 \\
\hline & Ortodoxa & 1.299 & 13 & 19 \\
\hline & Otra religión & 50.214 & 287 & 1.390 \\
\hline & Ninguna, ateo, agnóstico & 77.311 & 128 & 872 \\
\hline
\end{tabular}


de AM que viven en residencias 60,9\% son mujeres; respecto a su estado conyugal, $21 \%$ está casado, $35 \%$ soltero y $42 \%$ viudo; refieren saber leer y escribir 83,4\%; $15 \%$ se define como lisiado o presentan parálisis; presentan deficiencia mental $13 \% ; 67,6 \%$ refieren no tener discapacidad física y se presentan los resultados de las otras discapacidades (ceguera, sordera, mudez) que incluye la boleta del censo; con respecto a su ingreso económico 47\% es jubilado o rentista y $17 \%$ está incapacitado permanentemente para trabajar; la gran mayonía (81\%) dice profesar la religión católica.

$\mathrm{Al}$ analizar los resultados sobre la presencia de alguna limitación o el número de discapacidades, en el total de AM chilenos, se observa que la gran mayoría que las tiene o padece no están institucionalizados. Encontramos que vivirían en residencias sólo $6,1 \%$ de los lisiados o con parálisis, $18,9 \%$ de los con deficiencia mental, 9,1\% de los con dos y tres discapacidades, $16 \%$ de los con cuatro discapacidades y $2,7 \%$ de los con cinco discapacidades. También destaca, que 4,5\% del total de AM chilenos son solteros, pero aumenta a $35 \%$ en los AM institucionalizados.

\section{DisCUSIÓN}

El envejecimiento influye en todas las estructuras de la sociedad y produce una necesidad de servicios de cuidado adecuado para los adultos mayores (AM). Hasta ahora, el interés de los gobiernos por desarrollar servicios médicos para el AM ha sido lento y escaso. Se tiende a decir que el problema del anciano es social y se desestiman los informes que señalan que los tres problemas más relevantes para ellos, según importancia, son: mala salud, soledad y pobreza. En el hecho, en muchos países no existen servicios, programas estructurados de cuidado, ni capacitación del personal de salud y de los que toman decisiones para afrontar correctamente el desafío del envejecimiento y sobre todo del incremento de AM institucionalizados ${ }^{1-7}$.

Nuestros países deben enfrentar ahora este desafío y brindar servicios médicos-sociales a los AM y es por ello que se realizó en la CEPAL en Santiago (noviembre 2003) la «Conferencia Regional Intergubernamental sobre Envejecimiento: hacia una estrategia regional de implementación para América Latina y el Caribe del Plan de Acción Internacional de Madrid sobre el Envejecimiento», donde uno de los temas abordados en el documento de acuerdo fue la importancia que tiene el cuidado, a largo plazo en la región, y la pobre información que se dispone al respecto y ello motivó el presente estudio.

Los datos del Censo de 2002 demuestran que los mayores de 60 años son $11,4 \%$ de la población total y 670.000 habitan en la Región Metropolitana $(\mathrm{RM})$. Mientras la población total creció a tasas de $1,2 \%$, la de los AM fue de 3,3\% y se proyecta que en el año 2034 el número de AM será igual al de los menores de 15 años. Chile ya tiene la expectativa de vida más larga de Latinoamérica (78 años) y contamos con alguna información validada sobre el perfil de salud de los que viven en la comunidad ${ }^{16}$.

El tema de los «cuidados a largo plazo»por su relevancia actual, incremento en su uso y los costos proyectados ha sido abordado previamente por los países más desarrollados ${ }^{4-11}$. La mayoría se realiza en la vivienda, pero un porcentaje requiere vivir en instituciones. Los factores de riesgo de institucionalizarse son: limitación funcional severa, vivir solo, haber estado previamente viviendo en residencias, demencia con cambios conductuales, incontinencia de orina y agotamiento del cuidador ${ }^{7,9-11}$. El porcentaje de AM en instituciones es variable, así es como en la década 1980-1990 eran: Suiza 9,6\%, Canadá 8,6\%, Islandia 8\%, Australia 6,4\%, Francia 6,3\%, EEUU de NA $5,7 \%$, Reino Unido 5,1\%, Dinamarca 4\%, Japón $3,9 \%$ y Holanda 3,5\%5-11. Estas cifras incluyen aquellos AM que están varios meses en rehabilitación antes de ser enviados a sus casas.

En los EEUU de NA, se estima que cerca de 6\% del total de AM está en una institución. Pero este porcentaje varía dependiendo de la edad $(<3 \%$ entre 65-74 años a $25 \%$ en los $\geq 85$ años), del sexo (\% es menor en varones), de la condición general (mayor en los más limitados) y de la etnia ( $<\%$ en afroamericanos). En las mujeres que fallecieron después de los 89 años, 70\% habían permanecido algún tiempo institucionalizadas ${ }^{5,7,11}$. Este cuidado es muy costoso para los países, por ejemplo en EEUU de NA (1995) se gastaron $\$ 70$ billones de dólares o el $8 \%$ del gasto de salud y se estimó que aumentarán en el futuro. Por ello, se han desarrollado distintas iniciativas para reducir los costos, 
manteniendo la calidad y ampliando la variedad del cuidado crónico, a través de un instrumento común de evaluación de los residentes y así comparar lo que ocurre en diferentes países ${ }^{4-8}$.

En todos los países desarrollados ha crecido el porcentaje de AM institucionalizados, lo que implica que aumentaron los más ancianos, aquellos con escaso apoyo social y los más frágiles ${ }^{4-11}$. Algo similar se supone en nuestros países latinoamericanos, ya que cada año aumentan las ofertas de hogares» en la prensa. En Chile, son regidas por el Reglamento de Establecimientos de Larga Estancia para el Adulto Mayor- ELEAM» (octubre de 1994) y son fiscalizadas por las Unidades de Profesiones Médicas de los distintos Servicios de Salud. Usando el proxy» descrito, se estiman que hay 1.668 instituciones, de los cuales 624 corresponden a la definición de residencias colectivas informales» $(37,4 \%)$ y 1.044 serían residencias colectivas formales». En Santiago habría 804 instituciones (14.178 AM), pero sólo 324 de ellas están acreditadas. El estudio realizado por el MINSAL (1992) Evaluación de la calidad de Hogares de Ancianos», concluyó que según los criterios para clasificar las 123 residencias del Servicio de Salud Oriente, el $28,5 \%$ era regular y $39 \%$ era no recomendable por presentar déficit importantes en la estructura y dinámica de funcionamiento. Más aún, se demostró que 80,8\% del personal que trabaja en ellos presenta actitudes neutras 0 desfavorables respecto a la vejez (31\% francamente malas) y nada hace suponer que estas cifras hayan mejorado actualmente. Algo similar fue reportado en México, donde la calidad de la atención es con frecuencia deplorable, incluso dentro de las privadas ${ }^{17}$. Deducimos que la ausencia de un catastro, de una normativa y de una supervisión adecuada ha permitido la proliferación de instituciones (formales e informales) no calificadas para brindar cuidados a AM necesitados en toda la región.

Nuestro estudio, al usar un sproxi», tiene limitaciones, por lo cual los resultados aquí presentados deben ser tomados con cautela y como una primera aproximación a la situación real de la institucionalización de AM en Chile. Sin embargo, dada la alta correlación estadística que tiene este indicador con el porcentaje de AM institucionalizados encontrado en otros países de la región que sí tienen este dato definido, consideramos que se trata de una aproximación bastante razonable del número de residencias existentes en Chile.

El conocer las posibles instituciones, nos permitió tener una caracterización de los 26.854 residentes (ver Tablas) que corresponden al 1,56\% del total de AM. La mayoría de ellos vive en «esidencias formales» (62,6\%), incrementando su uso a mayor edad (7,6\% en los $>90$ años) y es más frecuente en el caso de ser mujer (61\%) y en los solteros ( $34 \%$ vs $4,5 \%$ del total de AM) y en la RM están el 48,2\% de las residencias (un tercio informales), pero sólo la mitad están acreditadas a pesar que la emplean el 52,8\% del total. Entre los AM con discapacidades, el $15 \%$ de los lisiados/ parálisis y el $13 \%$ de los clasificados con deficiencia mental habitan en instituciones; ello implica que la mayoría son cuidados en sus casas.

Se proyecta que el número de residentes aumentará, más que el incremento puro de la población de AM en Chile, por lo cual debemos prepararnos para brindar «cuidados prolongados» no importando dónde, sino que de acuerdo a sus necesidades, al mismo tiempo fomentar el apoyo familiar para evitar su institucionalización. Sin embargo, por el envejecimiento poblacional siempre existirá un porcentaje de AM institucionalizados $\mathrm{y}$, más aún, se proyecta que su número aumentará. Por ello, es fundamental cuantificarlos y conocer en qué condiciones están, qué tipo de personal los atiende, cómo es su infraestructura, etc., de manera de poder asistirlos y guiarlos en aquellos puntos deficitarios. En este sentido, es necesario homologar las residencias de largo plazo para velar por un trato digno y mejorar la calidad de vida de los AM residentes.

Finalmente, para cuantificar mejor el fenómeno, se requiere, por una parte, introducir en las futuras boletas censales las categorías separadas de viviendas colectivas de residencia para adulto mayor» $\mathrm{y}$ hospitales», al tiempo de hacer un catastro cada vez más completo de estas residencias. 


\section{REFERENCIAS}

1. Butler RN, Hyer K, Schechter M. The elderly in society: an international perspective. En: Brocklehurst JC, Tallis RC, Fillit HM, editores. Textbook of Geriatric Medicine and Gerontology. New York: Churchill Livingstone 1992; 980-92.

2. Department of Health. National Services Framework for Older People. London: UK, 2001.

3. MARín PP. La situación del adulto mayor en Chile (artículo especial). Rev Méd Chile 1998; 125: 1207-12.

4. HiRSCHFELD M, LINDSEY E. Community home-based care in resource-limited settings: a framework for action. WHO, 2002.

5. RiBBe MW. Nursing Homes in ten nations: a comparison between countries and settings. Age Ageing 1997; 26(S2): 3-12.

6. Brosky J, Habib J, Hirschfeld M. Key Policy issues in Long Term Care. The cross-cluster initiative on long term care, World Health Organization. WHO, 2003.

7. KANE RA, Kane RL. Long term care: principles, programs and policies. New York: Springer-Verlag, 1987.

8. Fries BE, Fahey CJ, Hirdes JP, Hawes C, Viadeck BC, MoRRIS J ET AL. Implementing the Resident Assessment Instrument: case studies of Policymaking for Long-Term Care in eight countries. Milbank Memorial Fund, May 2003. http://www.milbank.org/ reports/interRAI/ 030222interRAI.html»

9. GoLd G, MARCHELO V. Institutional Care. En: Evans G, Williams F, Beattie L, Miche JP, Wilcock GK, editores. Oxford Textbook of Geriatric Medicine. London: Oxford University Press, 2000; 1068-75.

10. Denham MJ. Long term care. En: Pathy JMS, editor. Principles and Practice of Geriatric Medicine. New York: John Wiley \& Sons, 1998; 1515-22.

11. Ouslander JG. The American nursing home. En: Brocklehurst JC, Tallis RC, Fillit HM, editores. Textbook of Geriatric Medicine and Gerontology. New York: Churchill Livingstone, 1992; 1000-4.

12. Marín PP, Castro S, Galeb I, Valenzuela E, Hoyl T. Estudio comparativo de nonagenarios que viven en sus propios hogares versus los institucionalizados. Rev Méd Chile 1998; 126: 761-8.

13. Marín PP, Kornfeid R, Somiai E, Valenzueia E, CASTRO S. Valoración geriátrica ambulatoria de 2.116 adultos mayores pobres. Rev Méd Chile 1998; 126: 609-14.

14. Marín PP, Gac H, Hoyl T, Carrasco M, Duery P, Cabezas M et al. Estudio comparativo de mujeres mayores de noventa años institucionalizadas. Rev Méd Chile 2004; 132: 33-9.

15. Censo de Población y Vivienda. Instituto Nacional de Estadística. Chile, 2002.

16. Perfil del Adulto Mayor en Chile. Desarrollando respuestas integradas de Sistemas de Cuidados de Salud para una población de rápido envejecimiento (INTRA). Publicación Ministerio de Salud y OPS-OMS, 2002.

17. GutiérRez LM, Reyes G, Rocabado F, López J. Evaluación de instituciones de cuidados prolongados para ancianos en el Distrito Federal. Una visión crítica. Salud Pública Méx 1996; 38(6): 487500 . 